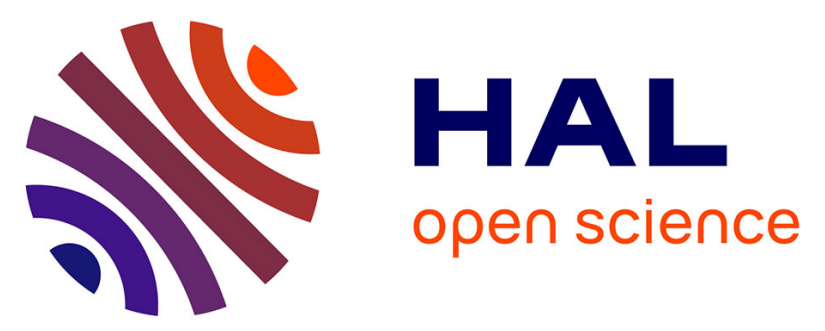

\title{
Comparaison de l'efficacité de cinq pièges non vulnérants à l'égard de la capture du Campagnol provençal (Pitymys duodecimcostatus de Sélys-Longchamps, 1839)
}

Gérald Guedon, M. Bélair, Michel Pascal

\section{- To cite this version:}

Gérald Guedon, M. Bélair, Michel Pascal. Comparaison de l'efficacité de cinq pièges non vulnérants à l'égard de la capture du Campagnol provençal (Pitymys duodecimcostatus de Sélys-Longchamps, 1839). Mammalia, 1992, 54 (1), pp.137-145. 10.1515/mamm.1990.54.1.137 . hal-02194747

\author{
HAL Id: hal-02194747 \\ https://hal.science/hal-02194747
}

Submitted on 25 Jul 2019

HAL is a multi-disciplinary open access archive for the deposit and dissemination of scientific research documents, whether they are published or not. The documents may come from teaching and research institutions in France or abroad, or from public or private research centers.
L'archive ouverte pluridisciplinaire HAL, est destinée au dépôt et à la diffusion de documents scientifiques de niveau recherche, publiés ou non, émanant des établissements d'enseignement et de recherche français ou étrangers, des laboratoires publics ou privés. 


\title{
Comparaison de l'efficacité de cinq pièges non vulnérants à l'égard de la capture du Campagnol provençal (Pitymys duodecimcostatus de Sélys-Longchamps, 1839)
}

\author{
par G. GUÉDON ', M. BÉLAIR ${ }^{2}$ et M. PASCAL ${ }^{2}$ \\ ${ }^{\prime}$ A.C.T.A., F.N.G.P.C., 936, rue d'Alco, B.P. 7309, F-34083 Montpellier Cedex \\ ${ }^{2}$ Laboratoire de la Faune Sauvage du Centre I.N.R.A. de Jouy-en-Josas, \\ F-78350 Jouy-en-Josas
}

Summary. - Comparative efficacity of five traps in the capture of Pitymys duodecimcostatus: Longworth, Sherman, I.N.R.A., Trip-Trap and Belair.

Résumé. - Les auteurs comparent l'efficacité, dans la capture de Pitymys duodecimcostatus, de cinq types de pièges non vulnérants : Longworth, Sherman, I.N.R.A., TripTrap et Bélair.

\section{INTRODUCTION}

La manipulation en nature de sujets vivants est souvent indispensable à l'étude de nombreux aspects de la biologie des populations de rongeurs. Si la mise au point de pièges non vulnérants permettant de telles manipulations est largement avancée pour de nombreuses espèces, il n'en est pas de même pour le Campagnol provençal (Pitymys duodecimcostatus De Sélys-Longchamps, 1839). En effet, les rares publications en notre possession et relatives à ce sujet font état de résultats médiocres. C'est ainsi que Viegas (in Sezinando) et Sezinando (1982), à l'issue du test de trois types de pièges, en retiennent deux donnant des résultats encourageants. Cependant, le premier, un piège tube à porte poussante, présente l'inconvénient de permettre un fort taux d'évasion et le second dont la fermeture à guillotine palie cet inconvénient, voit son système de déclenchement très fréquemment bloqué en raison du comportement de "bourrage " du rongeur, comportement souvent développé chez les espèces souterraines qui consiste à obstruer de terre toute ouverture pratiquée sur le réseau de galeries.

Une expérimentation a été mise en place dans le but de comparer l'efficacité de capture du Campagnol provençal par cinq types de pièges non vulnérants à celle d'un piège vulnérant donnant satisfaction (Guédon 1987).

Mammalia, t. 54, $n^{\circ} 1,1990$. 

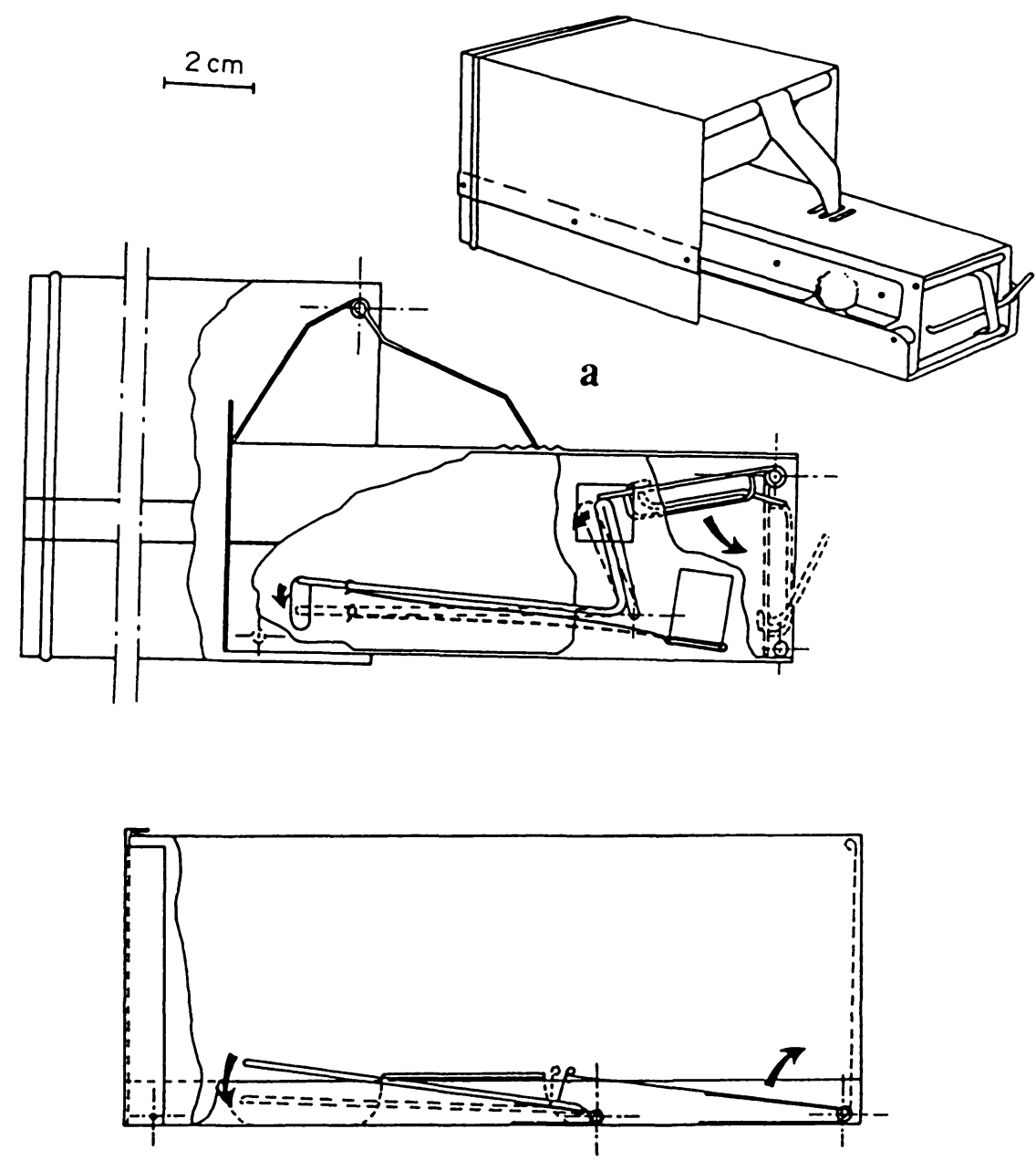

b

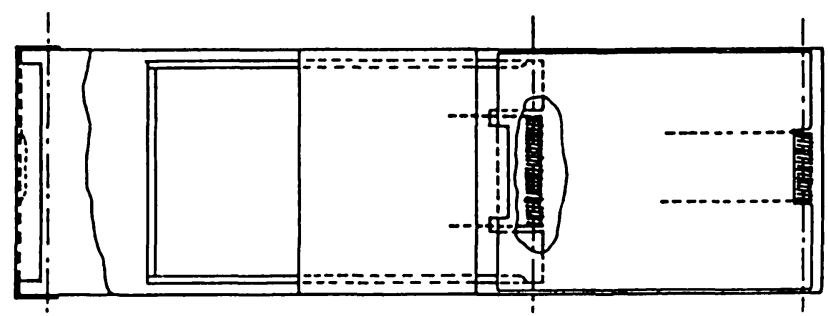

Fig. 1. - Plans cotés des pièges Longworth (a), Sherman (b), I.N.R.A. (c), Trip-Trap(dl), Bélair (e) et pince (f). 


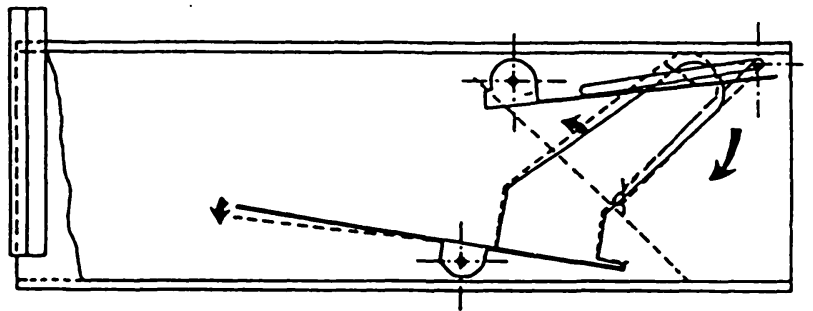

c
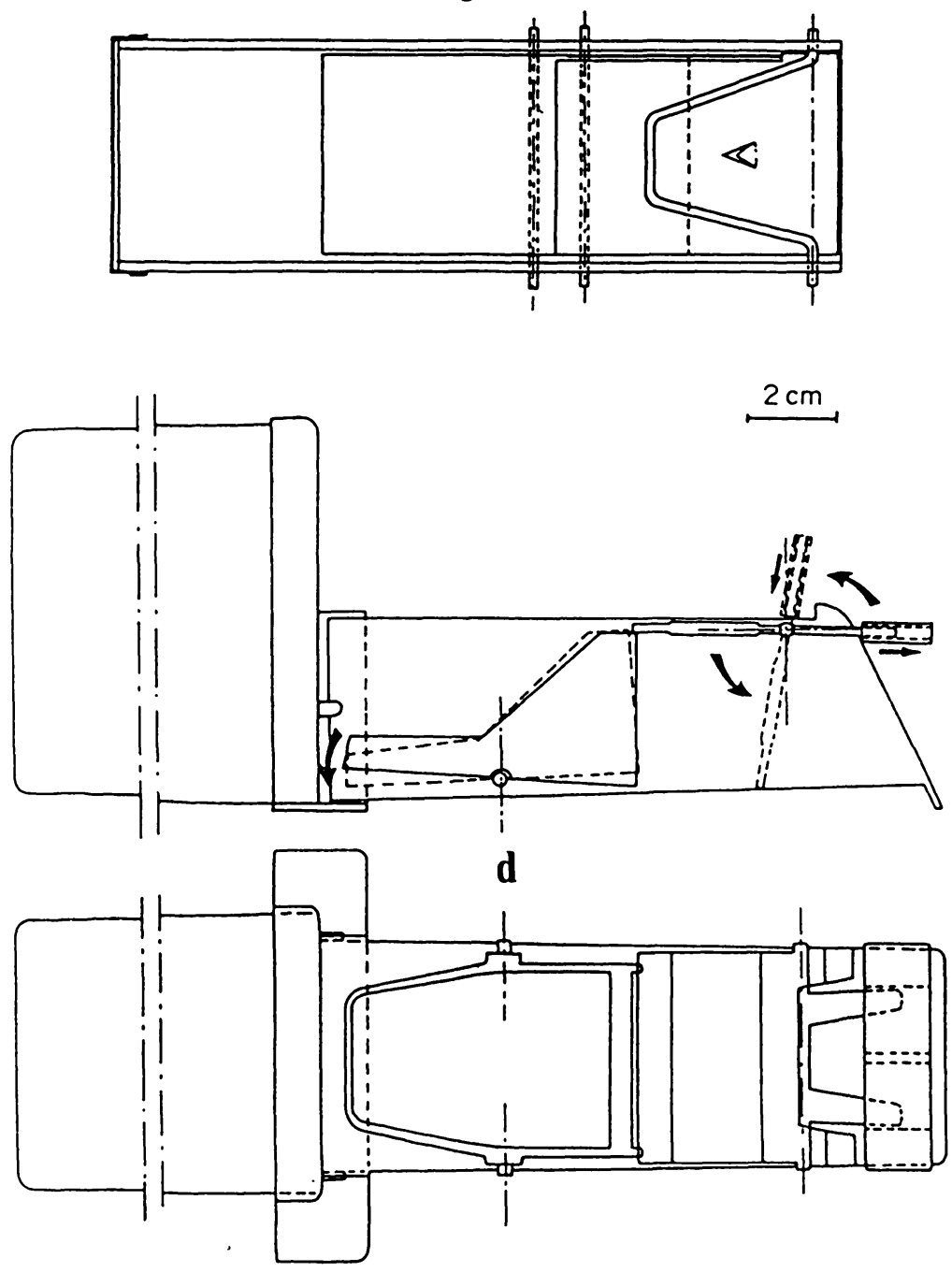


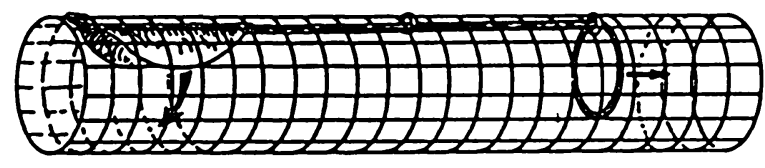

$\mathbf{e}$
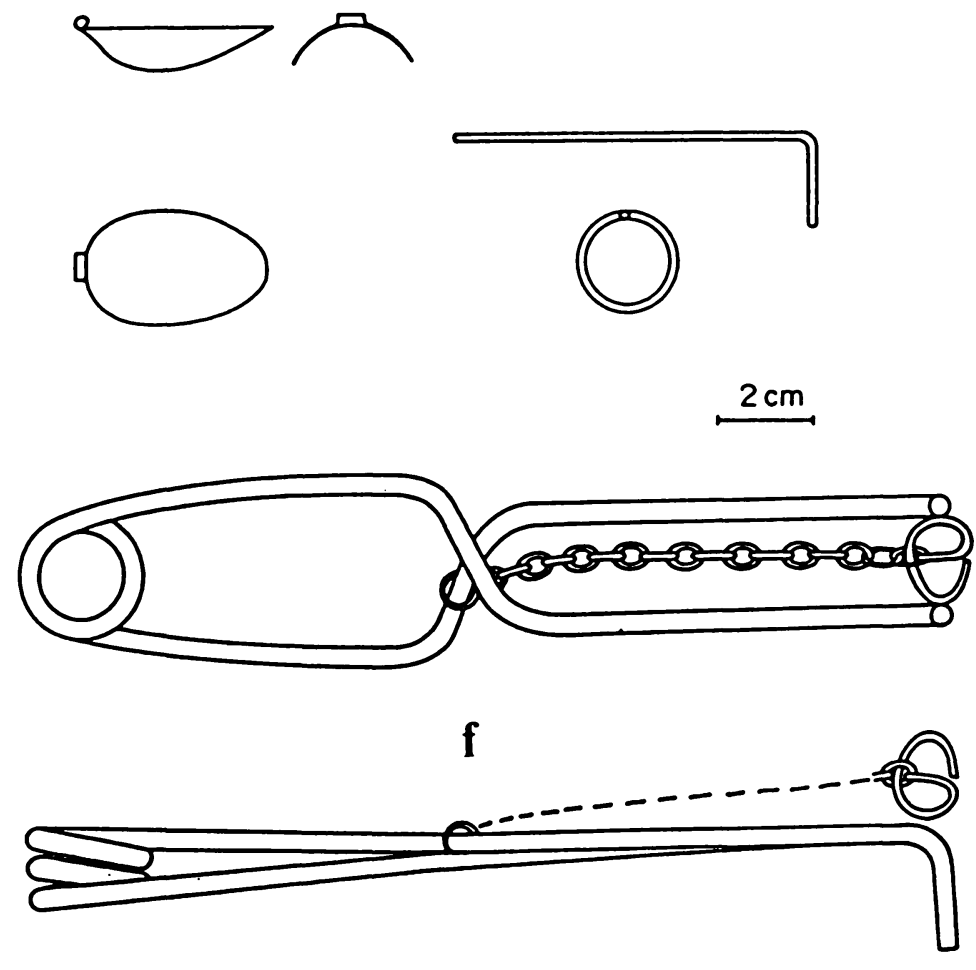

MATÉRIEL ET MÉTHODE

1) Matériel de capture.

Parmi les cinq types de pièges non vulnérants, quatre sont vendus dans le commerce (Annexe 1). Il s'agit du piège Longworth (Fig. 1 a), utilisé par Salvioni (1986) pour l'étude des déplacements de $P$. multiplex, $P$. savii et $P$. subterraneus, du petit Sherman (Fig. 1b), employé lors de nombreuses études portant sur la biologie de $P$. pinetorum (Cornbower et Kirkland, 1983 ; Fitzgerald et Madison, 1983 inter alia), du piège Le Chapellier et Aubry (Aubry 1950) ou piège I.N.R.A. (Fig. 1c), utilisé, entre autres, par Spitz (1974) pour l'étude de la biologie de Microtus arvalis, espèce voisine du Campagnol provençal par la taille et du piège Trip-Trap (Fig. 1d) récemment commercialisé.

Le cinquième type de piège, le piège Bélair (Fig. 1e), a été conçu conjointement par l'I.N.R.A. et l'A.C.T.A. Une série de mensurations pratiquées sur 
le rongeur (diamètre du crâne, longueur du corps) et ses galeries (diamètre) ont servi à établir respectivement la longueur et le diamètre du piège.

Le piège vulnérant de référence (Fig. $1 \mathrm{f}$ ) est dérivé du piège pince utilisé, entre autres, pour l'étude de la biologie d'Arvicola terrestris (Pascal et Meylan 1986). Les modifications apportées à l'original consistent en une réduction de la hauteur des mors pour l'adapter au plus faible diamètre des galeries du Campagnol provençal et en une augmentation de sa longueur générale afin de palier, au moins partiellement, les conséquences du comportement de bourrage du rongeur.

\section{2) Mise en place de l'expérimentation.}

L'expérimentation a été implantée sur un verger de pommiers de 6 ha (commune de Mudaison, Hérault, France), relativement isolé du milieu environnant par de larges chemins d'exploitation (Fig. 2) et abritant une population apparemment importante et régulièrement répartie de campagnols provençaux.

En raison du nombre réduit de certains types de pièges (10 unités), il a été procédé à quatre répétitions successives (A : novembre 1987 ; $\mathrm{B}$ : janvier 1988 ; C et D : février 1988) d'une même expérience élémentaire (Fig. 2).

A l'occasion de chaque expérience élémentaire, les cinq types de pièges non vulnérants et le piège pince vulnérant sont utilisés simultanément, les premiers sur une seule unité de piégeage, le second sur quatre.

Chaque unité de piégeage est installée sur cinq carrés de $5 \mathrm{~m} \times 5 \mathrm{~m}$ délimitant une bande de $5 \mathrm{~m} \times 25 \mathrm{~m}$. Sur chaque carré, deux emplacements sont choisis en fonction de signes extérieurs de présence du rongeur et reçoivent chacun un piège (parfois plusieurs pour le seul piège pince). Les diverses unités de piégeage de chaque expérience élémentaire sont placées dans le prolongement les unes des autres délimitant ainsi une longue bande de terrain de $5 \mathrm{~m}$ de large. Leurs emplacements relatifs sur cette bande sont tirés au hasard, exception faite des unités de pièges vulnérants qui sont systématiquement rejetées aux extrémités afin de mettre en évidence une éventuelle hétérogénéité de la densité du rongeur sur le site. En raison des caractéristiques géométriques de la parcelle expérimentale et de l'orientation générale des bandes, perpendiculaire à l'orientation des lignes d'arbres (Guédon 1987), les unités de piégeages de chaque expérience élémentaire ont été réparties sur deux bandes (Fig. 2).

Les pièges, non appâtés, sont placés, après ouverture du terrier, soit directement dans la galerie (piège pince et piège Bélair), soit en dérivation, perpendiculairement à son axe. Ils sont contrôlés huit fois sur une période de 48 heures, l'intervalle de temps minimum entre deux contrôles étant de 3 heures. A l'occasion de chaque relevé, l'emplacement de chaque capture et les manifestations du comportement de bourrage sont soigneusement consignés. Suivant la méthode décrite par Pascal et Meylan (1986), lors du dernier contrôle, une synthèse relative à ce comportement est réalisée afin d'évaluer le nombre minimum de rongeurs ayant échappé au piégeage (B).

L'efficacité du piégeage a été évaluée par deux indices. Le premier est le nombre de captures $(N)$ par unité de piégeage $(N / U)$ : il croît avec l'efficacité du piège mais n'intègre pas une évaluation du nombre d'individus potentiellement capturables sur le site de l'unité de piégeage ; le second tient compte de l'insuccès de capture lié au comportement de bourrage du rongeur $(B / U)$ et décroît avec l'efficacité du piégeage. 


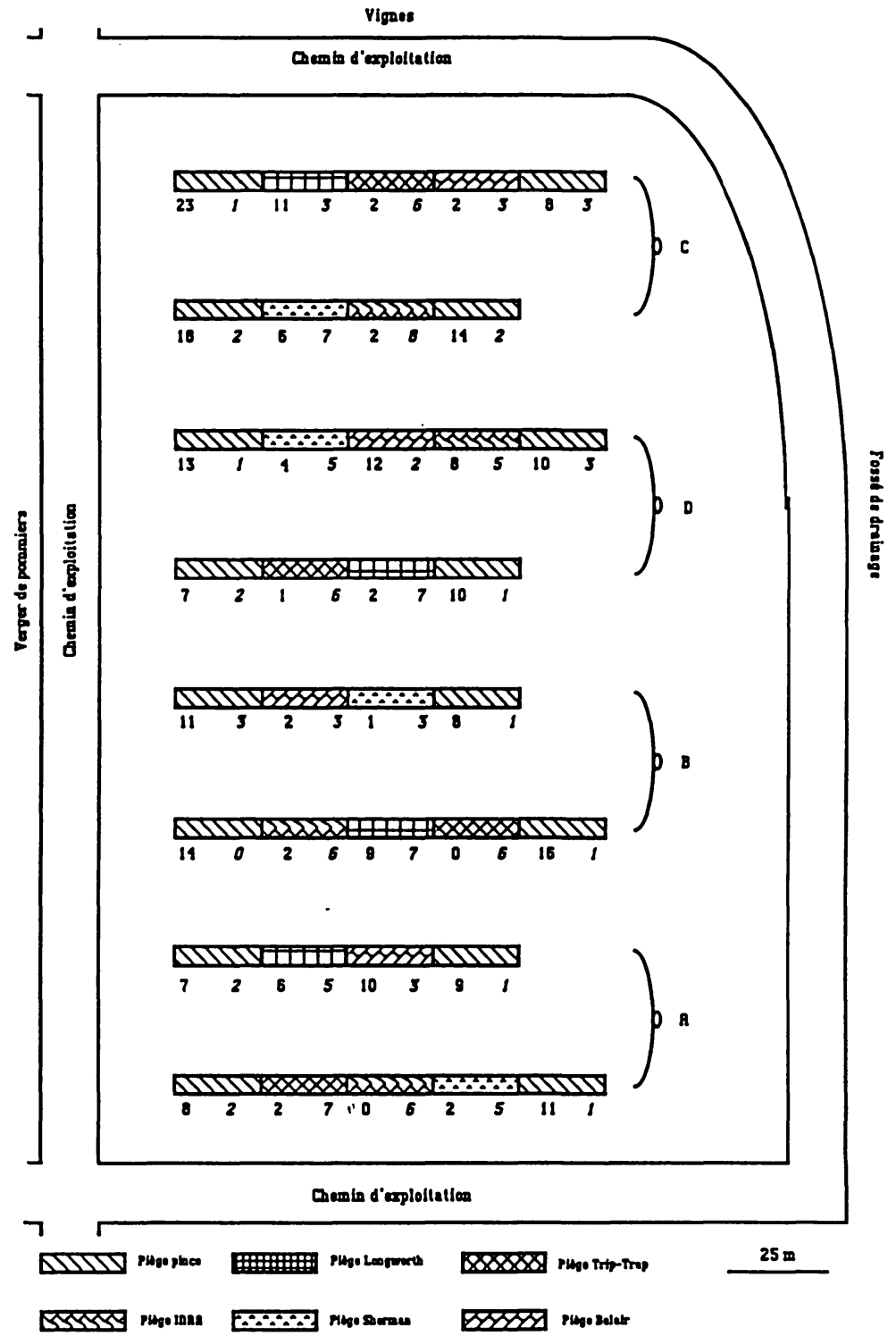

Fig. 2. - Plan d'implantation des diverses expériences.

\section{RÉSULTATS}

L'ensemble des résultats relatifs au nombre de captures et de manifestations de bourrage est consigné dans la figure 2. L'examen global des données monttre que le piège pince est substantiellement plus efficace que les autres (Tab. 1 ), 
tant par la valeur élevée du nombre de captures par unité de piégeage que par celle, très basse, du taux d'insuccès de captures. Il est vrai que les postes de piégeage équipés de pièges pinces peuvent en comporter plusieurs alors qu'ils n'en comportent qu'un dans le cas des pièges non vulnérants. Cependant, ce fait ne peut à lui seul expliquer la meilleure efficacité du piège pince. En effet, à condition que la durée du piégeage ne soit pas excessive, la multiplication du nombre de piège en un même point d'un réseau de galeries augmente la vitesse des captures mais pas leur nombre (Pascal et Meylan 1986), la proportion d'évitement peut être considérée comme inchangée. Par ailleurs, les divers types de pièges non vulnérants se répartissent en trois catégories d'intérêt décroissant. La première englobe le piège Longworth et le piège Bélair, la seconde les pièges Sherman et I.N.R.A., la troisième ne comporte que le piège Trip-Trap, de loin celui qui donne les résultats les plus décevants. Cependant, cet ensemble de conclusions pourrait se révéler erroné si la répartition densitaire du rongeur sur les diverses unités de piégeage n'était pas "raisonnablement " homogène.

La recherche d'une éventuelle hétérogénéité spatiale de la densité du rongeur sur l'ensemble de la parcelle a été réalisée en ne tenant compte que des résultats relatifs aux seize unités de piégeage mettant en œuvre le piège pince. La somme du nombre d'individus capturés $(\mathrm{N})$ et du nombre de manifestations de bourrage (B) constitue le meilleur indice disponible rendant compte de la présence du rongeur. La comparaison par un test de $\chi^{2}$ des seize valeurs de cet indice à la valeur moyenne de l'indice ne permet pas de mettre en évidence une hétérogénéité spatiale du peuplement $\left(\chi_{15}^{2}\right.$ ddl $\left.=20,38\right)$.

Afin de déterminer si le comportement du piège pince restait similaire d'une unité de piégeage à l'autre, soit donc à diverses dates, nous avons comparé, pour chaque expérience élémentaire, le nombre de captures réalisées $(\mathrm{N})$ au nombre de bourrages observés (B) au moyen d'un test de $\chi^{2}$. Les résultats de ces tests $\left(\chi^{2}{ }_{3 \text { ddl }}\right.$ est égal pour l'expérience $\left.\mathrm{A}: 4,09 ; \mathrm{B}: 2,02 ; \mathrm{C}: 4,16 ; \mathrm{D}: 1,20\right)$ ne permettent pas de conclure à une hétérogénéité de comportement du piège pince d'une expérience élémentaire à l'autre.

TABLEAU 1. - Résultats expérimentaux. $\Sigma U$ : Nombre d'unités de piégeage. $\Sigma \mathrm{N}$ : Nombre de captures, $\Sigma \mathrm{B}:$ Nombre de postes de piégeage bourrés de terre par le rongeur, $\Sigma \mathrm{N} / \Sigma \mathrm{\Sigma}$ : Nombre moyen de captures par unité de piégeage, $\Sigma B / \Sigma U$ : Nombre moyen de bourrages par unité de piégeage.

\begin{tabular}{ccccccc} 
& Pince & Longworth & Belair & Sherman & INRA & Trip-Trap \\
\cline { 2 - 7 }$\sum U$ & 16 & 4 & 4 & 4 & 4 & 4 \\
$\Sigma N$ & 187 & 28 & 26 & 13 & 12 & 5 \\
$\Sigma B$ & 26 & 22 & 11 & 21 & 25 & 25 \\
$\Sigma N \sqrt{\Sigma} U$ & 11,7 & 7 & 6,5 & 3,3 & 3 & 1,3 \\
$\sum B / \Sigma U$ & 1,63 & 5,50 & 2,75 & 5,25 & 6,25 & 6,25
\end{tabular}

Le classement des six types de pièges en fonction du nombre de captures positionne le piège pince en première place pour chacune des quatre expériences élémentaires. Sous l'hypothèse qu'il n'y a pas de différence de performance entre les types de pièges, ce résultat, ou un résultat aussi ordonné, est hautement improbable $\left((1 / 6)^{4} \times 2=1,54 \times 10^{-3}\right)$. Le positionnement systématique du 
piège pince en première place peut donc être raisonnablement attribué à sa meilleure performance.

L'analyse globale (Tab. 1) distingue deux groupes de pièges aux performances apparemment différentes : d'une part le Sherman et l'I.N.R.A., d'autre part le Longworth et le Bélair. Nous avons comparé au moyen d'un test de Student la valeur moyenne du nombre de captures obtenue par l'un et l'autre groupe de pièges. Il apparaît que le nombre moyen de captures réalisé par l'ensemble des pièges Longworth et Bélair est significativement plus élevé que celui réalisé par les pièges Sherman et I.N.R.A. $\left(t_{14} \mathrm{ddl}=2,02 ; p<0,05\right)$. En revanche, le nombre moyen de pièges bourrés n'est pas significativement plus faible pour les premiers que pour les seconds $\left(t_{14 d a l}=1,72 ; p<0,2\right)$.

Si le nombre moyen de captures réalisé par le piège Bélair n'est pas significativement différent de celui obtenu par le piège Longworth $\left(t_{6 \text { ddl }}=0,15 ; p<0,9\right)$, en revanche, le nombre moyen de pièges bourrés associé aux pièges Longworth est très significativement plus élevé que celui associé aux pièges Bélair $\left(t_{6 d d l}=2,78 ; p<0,05\right)$.

\section{CONCLUSIONS - DISCUSSION}

Il n'a pas été possible de mettre en évidence d'une part une hétérogénéité de la densité du Campagnol provençal sur l'ensemble de la parcelle expérimentale, d'autre part, une variabilité du comportement du rongeur à l'égard du piège pince au gré des diverses expériences. Ces résultats justifient, en première analyse, de comparer l'efficacité des divers types de pièges les uns par rapport aux autres.

Le piège pince se distingue des cinq types de pièges vulnérants par une meilleure efficacité de piégeage qui se manifeste tant par un fort taux de capture que par un faible taux de bourrage.

TABLEAU 2. - Classement en fonction de cinq critères et selon trois niveaux (par ordre d'intérêt décroissant, de trois à une astérisques) des cinq types de pièges non vulnérants testés dans cette expérimentation.

\begin{tabular}{|c|c|c|c|c|c|}
\hline Piège & Efricacitè & Pose & Nettoyege & Côut & Encombrement \\
\hline Longworth & $* * *$ & $* * *$ & $\ldots$ & * & - \\
\hline Belair & $* * *$ & - & - & $* *$ & ** \\
\hline shermon & $*$ & $* *$ & $* *$ & $*$ & $*$ \\
\hline INRA & ** & $* * *$ & $\cdots$ & $*$ & $* *$ \\
\hline Trip-Trap & $*$ & ** & * & $*$ & * \\
\hline
\end{tabular}

Les pièges Sherman et I.N.R.A. se révèlent significativement moins efficaces que les pièges Longworth et Bélair. Ce dernier se montrerait aussi efficace que le piège Longworth et significativement moins sensible aux manifestations de bourrage. A ce stade de l'analyse, il convient cependant d'être prudent en raison du faible nombre de répétitions des expériences et de faire intervenir dans un 
éventuel argumentaire de choix des critères difficilement quantifiables (difficultés de pose ou de nettoyage) ou sans rapport direct avec l'efficacité proprement dite (prix) (Tab. 2).

\section{BIBLIOGRAPHIE}

AUBRY, J., 1950. - Deux pièges pour la captures de petits rongeurs vivants. Mammalia, 14 : 174-177.

Cornbower, T.R. et G.L. KirkLAND, 1983. - Comparisons of pine vole (Pitymys pinetorum) populations from orchards and natural habitation southcentral Pennsylvania. Proceedings of the Pennsylvania Academy of Science, 57 : 147-154.

FitzGerald, R.W. et D.M. MAdison, 1983. - Social organization of a free-ranging population of pine voles Microtus pinetorum. Behavioural Ecology Sociobiology, 13 : 183-187.

GuÉDON, G., 1987. - Influence de diverses spéculations et pratiques culturales sur certains paramètres de la cinétique de population du Campagnol à douze côtes, Pitymys duodecimcostatus (De Sélys-Longchamps, 1839) dans le Montpellierais (France). D.E.A. Sciences de l'Evolution et Ecologie. U.S.T.L. Montpellier : 29 pp.

Pascal, M. et A. Meylan, 1986. - L'échantillonnage linéaire des populations de la forme fouisseuse du Campagnol terrestre (Arvicola terrestris scherman (Shaw)). La Défense des Végétaux, 237 : 3-12.

SAlvioni, M., 1986. - Domaines vitaux, relations sociales et rythme d'activité chez trois espèces de Pitymys (Mammalia, rodentia). Thèse de docteur ès Sciences. Université de Lausanne : $135 \mathrm{pp}$.

SEZINANDO, T.I.E., 1982. - Contribuçao para o estudo bioecologico do rato toupeira, Pitymys duodecimcostatus (De Sélys-Longchamps, 1839), na regiao do algarve. Estagiaro do curso de engenheiro agronomo. Lisboa: $125 \mathrm{pp}$.

SPITZ, F., 1974. - Démographie du Campagnol des champs, Microtus arvalis, en Vendée. Ann. Zool. Ecol. Anim., 6 : 259-312.

\section{ANNEXE 1}

Le prix unitaire des divers types de pièges est exprimé en francs français T.T.C. hors frais de transport et droit de douane.

- Piège I.N.R.A. - B.T.S., 41, chemin des Torcols, F-25000 Besançon. Prix : 64,63 FF.

- Piège Sherman - H.B. Sherman Traps Inc. P.O. BOX 20267 Tallahassee, Florida 32336, U.S.A. Prix : 51,01 FF.

- Piège Trip-Trap - North West Plasyics Ltd. Mosley Common Road. Worsley Manchester M28 4AJ, G.B. Prix : 49,94 FF.

- Piège Longworth - Penslon Ltd. Abingdon OX14 3PH, England. Prix : 49,94 FF.

- Piège Bélair - B.T.S., 41, chemin des Torcols, F-25000 Besançon. Prix : 20 FF sur estimation de la production de près-série.

- Piège pince Pitymys - B.T.S., 41, chemin des Torcols, F-25000 Besançon. Prix : 3 FF. 
Brought to you by | INRA Institut National de la Recherche Agronomique Authenticated Download Date | 7/25/19 2:15 PM 